\title{
CORIORRETINOPATÍA SEROSA CENTRAL CON EXUDADOS SUBRETINIANOS BILATERALES
}

\author{
BILATERAL CENTRAL SEROUS CHORIORETINOPATHY \\ WITH SUBRETINAL EXUDATION
}

\author{
NORMAND-DE-LA-SOTILLA E ${ }^{1}$, CAMACHO-SAMPELAYO JM ${ }^{1}$, ROMERO-GUERRERO F ${ }^{1}$, \\ MUIÑOS-MURO S ${ }^{2}$
}

\section{RESUMEN}

Caso clínico: Varón de 37 años con disminución de agudeza visual bilateral y metamorfopsia. Presenta exudados subretinianos bilaterales amarillentos con levantamiento neurosensorial en el polo posterior, que en la angiografía se corresponden con puntos hiperfluorescentes en fases precoces. Tratado con fotocoagulación de los puntos de fuga, a los 6 meses recuperó su agudeza visual con una remisión prácticamente completa de las lesiones.

Discusión: La exploración clínica, las pruebas complementarias y la evolución del cuadro nos permiten descartar el diagnóstico de enfermedad de Best. La presencia de exudados subretinianos amarillentos no nos debe hacer desechar de entrada el diagnóstico de coriorretinopatía serosa central.

Palabras clave: Coriorretinopatía serosa central, angiografía fluoresceínica, tomografía de coherencia óptica.

\begin{abstract}
Clinical case: A 37-year-old man was referred because of a bilateral loss of visual acuity and metamorphopsia. On examination there was yellowish exudation bilaterally associated with serous retinal detachments, corresponding to hyperfluorescence spots in the early stage of fluorescein angiography. Photocoagulation of the leakage spots was performed in both eyes. Six months later he had recovered his visual acuity and the lesions had remitted.

Discussion: The clinical findings, ancillary tests and subsequent clinical course allowed a diagnosis of Best's disease to be ruled out. The presence of sub-retinal yellowish exudation in the posterior pole does not necessarily rule out the diagnosis of central serous chorioretinopathy (Arch Soc Esp Oftalmol 2006; 81: 595-598).
\end{abstract}

Key words: Central serous chorioretinopathy, fluorescein angiography, optical coherence tomography.

\section{INTRODUCCIÓN}

La coriorretinopatía serosa central (CSC) es una alteración corioretiniana idiopática caracterizada por un desprendimiento seroso de la retina neurosensorial en la región macular. A este desprendi- miento seroso pueden asociarse alteraciones del epitelio pigmentario (levantamiento del epitelio pigmentario, dispersión de pigmento) y exudación o depósitos subretinianos. La CSC puede manifestarse de forma uni o bilateral y presentar recurrencias.

\footnotetext{
Recibido: 30/5/05. Aceptado: 19/10/06.

Instituto Universitario Barraquer. Centro de Oftalmología Barraquer. Universidad Autónoma de Barcelona. Barcelona. España.

1 Licenciado en Medicina.

2 Doctor en Medicina.

Correspondencia:

Enrique Normand de la Sotilla

C/. García de Paredes, 66

28010 Madrid

España

E-mail: ens@inicia.es
} 


\section{CASO CLÍNICO}

Varón de 37 años, hipermétrope, con problemas visuales desde hacía 9 años, acudió a consulta por disminución de agudeza visual (AV) bilateral y metamorfopsia en ambos ojos (AO) tras un período de estrés. Su AV con sus gafas (+4,25 dioptrías en $\mathrm{AO}$ ) es de 0,5 en ojo derecho (OD) y 0,3 en ojo izquierdo (OI), aumentando la $\mathrm{AV}$ del OI a 0,85 con una corrección de $+7,00$ dioptrías.

A la exploración presenta exudados subretinianos bilaterales con levantamiento neurosensorial (LNS) del área macular (fig. 1). En la angiografía fluoresceínica (AGF) se observaron puntos hiperfluorescentes en polo posterior en fases precoces que aumentaban en intensidad y tamaño en fases inter- medias, asociados a un desprendimiento del epitelio pigmentario (DEP) (fig. 2). En la tomografía de coherencia óptica se apreciaron con mayor detalle el LNS y el DEP (fig. 3).

$\mathrm{Al}$ mes la AV del OD descendió a 0,3, por lo que se aplica fotocoagulación (FCG) con láser argón del punto de fuga en OD. A los dos meses, la lesión del OD se había reabsorbido y persistía la del OI. En este momento se decidió realizar una FCG en OI. Seis meses después del inicio del cuadro tan sólo se observa un punto de pigmento por la cicatrización del láser en $\mathrm{AO}$ (fig. 4) y la $\mathrm{AV}$ con corrección ( $+4,50$ dioptrías en $\mathrm{OD},+5,00$ dioptrías en $\mathrm{OI})$ era de 0,8 en AO. Durante dos años y medio de seguimiento no ha existido recurrencia de su enfermedad.

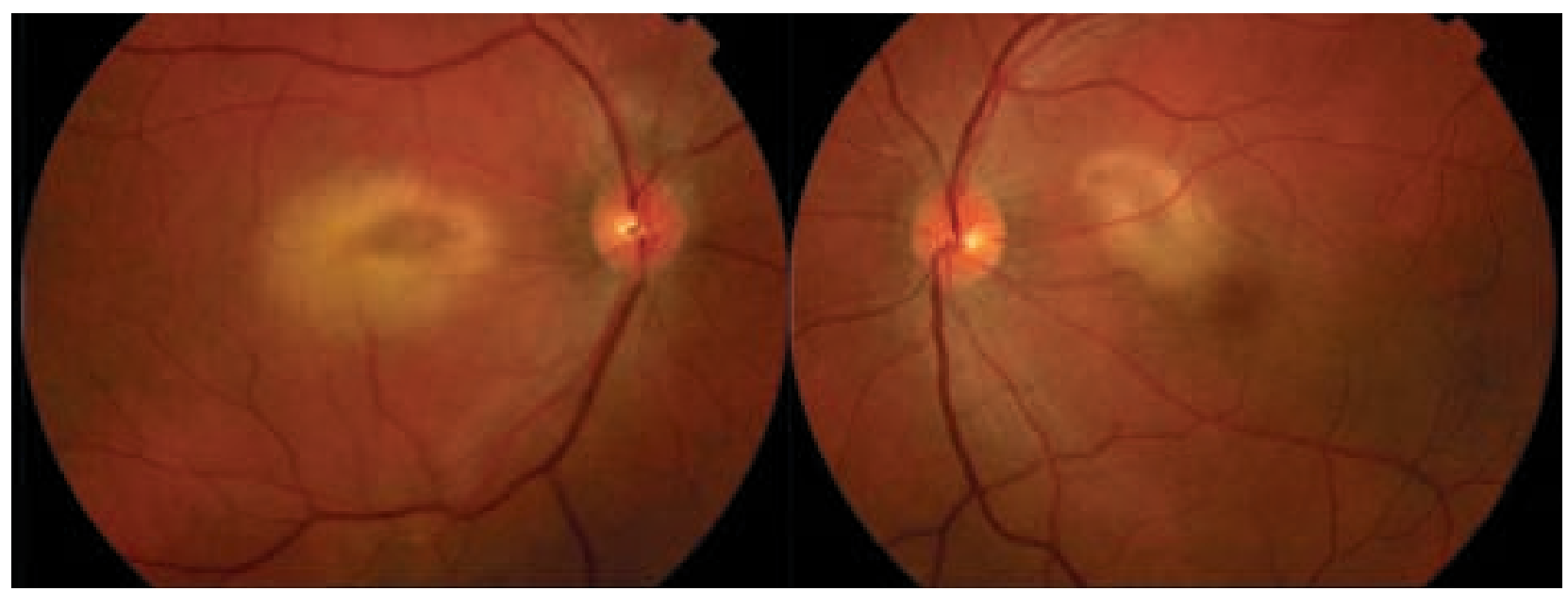

Fig. 1: Estado inicial del paciente. Exudados subretinianos bilaterales en polo posterior con levantamiento neurosensorial asociado.

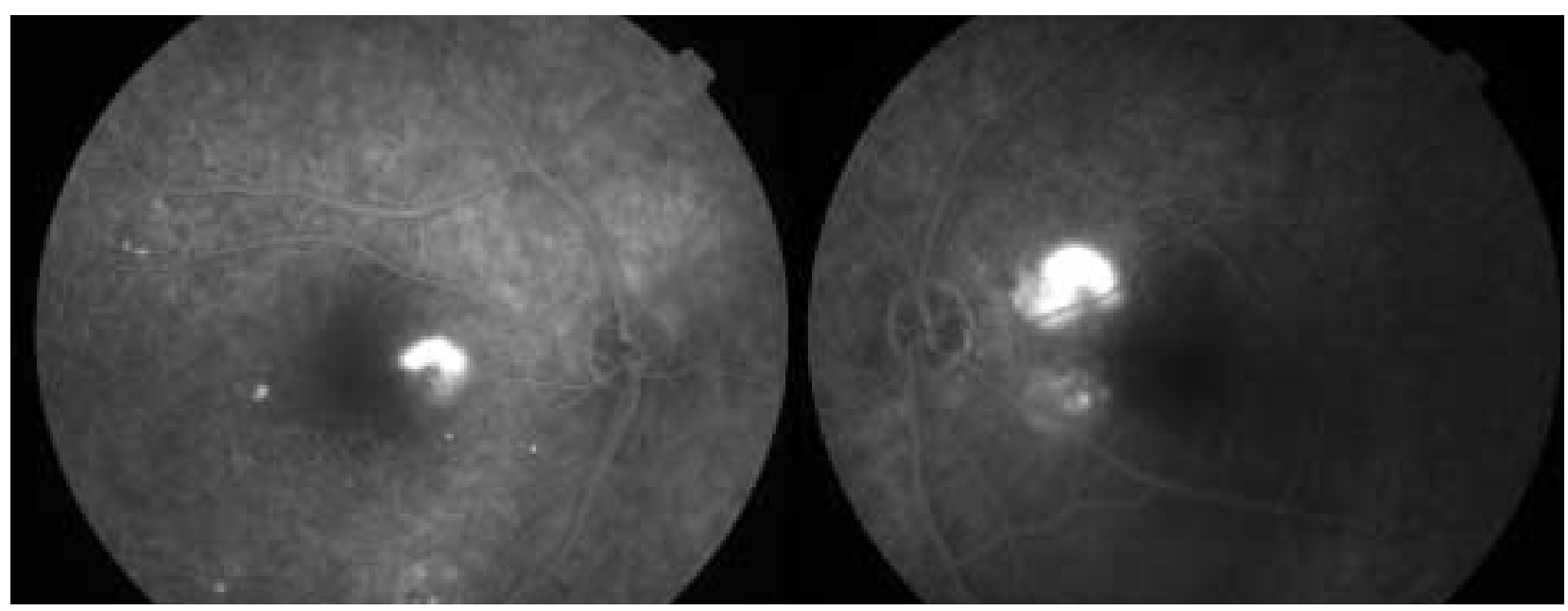

Fig. 2: Se observan puntos hiperfluorescentes en fases precoces del angiograma. 


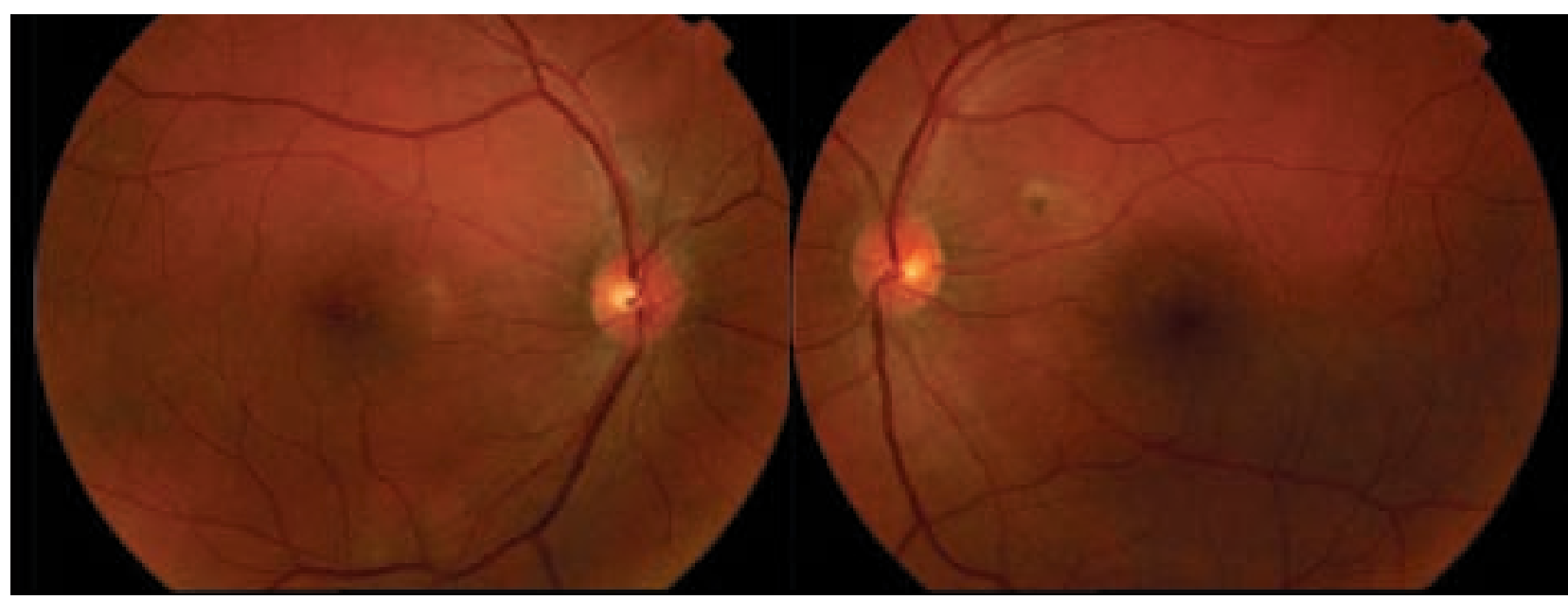

Fig. 4: Estado final del paciente. Se observa la cicatriz del impacto de fotocoagulación en OI.

\section{DISCUSIÓN}

Varios autores han descrito casos de coriorretinopatía serosa central (CSC) unilateral con una lesión viteliforme $(1,2)$. En la CSC también pueden encontrarse depósitos subretinianos blanquecinos y exudación lipídica $(3,4)$, similares a los que se presentan en este caso clínico.

La enfermedad de Best o distrofia foveomacular viteliforme del adulto es una entidad de herencia autosómica dominante que presenta varias fases en el examen oftalmoscópico. La AGF en este cuadro muestra un bloqueo de la fluorescencia coroidea por las lesiones viteliformes. Aunque no se consideró necesario la realización de un electrooculograma en este paciente, la exploración clínica, los hallazgos angiográficos y la evolución del cuadro nos permiten descartar el diagnóstico de enfermedad de Best.

Por tanto, la presencia de exudados subretinianos o de una lesión viteliforme en el polo posterior no nos debe hacer desechar de entrada el diagnóstico de CSC.

Actualmente, no existen evidencias de que la FCG mejore la AV final en los ojos afectos de CSC. Sin embargo, sí acorta el tiempo de evolución.

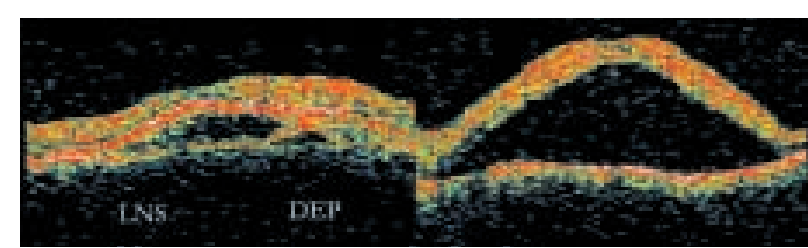

Fig. 3: La tomografía de coherencia óptica permite estudiar con mayor detalle el desprendimiento del epitelio pigmentario (DEP) en OD y el levantamiento neurosensorial (LNS) en AO.

\section{BIBLIOGRAFÍA}

1. Ikeda T, Sato K, Danjo Y, Tokuyama T, Ikeda N, Mimura $O$. Central serous chorioretinopathy exhibiting a vitelliform lesion similar to best disease. Arch Ophthalmol 2003; 121: 146-147.

2. Spaide RF. Deposition of yellow submacular material in central serous chorioretinopathy resembling adult-onset foveomacular vitelliform dystrophy. Retina 2004; 24: 301 304.

3. Iida T, Spaide RF, Haas A, Yannuzzi LA, Jampol LM, Lesser RL. Leopard-spot pattern of yellowish subretinal deposits in central serous chorioretinopathy. Arch Ophthalmol 2002; 120: 37-42.

4. Ie D, Yannuzzi LA, Spaide RF, Rabb MF, Blair NP, Daily MJ. Subretinal exudative deposits in central serous chorioretinopathy. Br J Ophthalmol 1993; 77: 349-353. 